\title{
Linking ATIS/ATMS and Environmental Plume Dispersion Models
}

\author{
Bruce Hellinga, Mark Baker, Mark Carter and Michel Van Aerde \\ Department of Civil Engineering, Ellis Hall, Queenș University, Kingston, Ontario, Canada K7L 3N6
}

\begin{abstract}
Recent legislation, which links federal funding with the successful demonstration that emission standards will be met, have caused traffic engineers to become increasingly concerned with the monitoring, control, and mitigation of air-borne pollutants produced by automobiles. In order to reduce the level of these emissions, many traffic engineers are considering the application of a variety of ATIS/ATMS strategies. Unfortunately, it is often difficult to determine the relative impact of one ITS strategy versus another, or of ITS strategies versus non-ITS approaches. As a result, traffic engineers have also begun to search for appropriate methods for estimating changes in the generation and dispersion of pollutants as a result of different ATIS/ATMS strategies.
\end{abstract}

The standard approach, to modelling emission dispersion, relies on the use of Air Quality Standard (AQS) models. However, these models generally require as input a spatially correlated time series of the magnitude of the pollutant input sources. An accurate estimate of this input data stream, or of changes to this input stream due to the deployment of ATIS/ATMS, has been to date difficult to obtain.

This paper describes the development and sample application of an extension to a standard traffic network modelling approach, that can be used to develop either the point, line or area source emissions that are required as inputs to AQS models. The application of this modelling approach is illustrated for a network representing Orlando, Florida to demonstrate its functional capabilities and its applicability to realistic sized urban centres.

\section{INTRODUCTION}

\section{A. Background and Objectives}

One of the prime motives, for deploying Advanced Traveler Information Systems (ATIS) and Advanced Traffic Management Systems (ATMS) strategies, is to mitigate the impact that automobiles have on air pollution in large urban areas. This ultimate impact is often a complex product of the temporal variation of the number of vehicles in the network, their speed characteristics as they encounter traffic congestion, their spatial distribution relative to the topography of the area.

To date, environmental engineers have developed extensive capabilities for modeling the temporal migration and dilution of air pollution from known sources. This migration is primarily a function of the prevailing terrain topography, the corresponding winds, and the interaction with sunlight and other airborne compounds. In contrast, transportation engineers have developed planning models for estimating the total cumulative weight of source production emissions, as a function of the total number of vehicle miles driven in the system.

What has been lacking, however, is the availability of a dynamic traffic model that can produce the spatial and temporal vehicle source emissions profile of an urban area during a peak period at a level of detail that is consistent with the needs of these atmospheric dispersion models.

This paper describes the development and application of an extension to a dynamic traffic model called INTEGRATION, which bridges this gap. The model can be utilized to develop the point, line, or area source emissions for use as inputs to a dispersion model. These inputs are sensitive to the temporal growth and decay of a dynamic traffic demand matrix during a typical peak period, and are based on a dynamic vehiclespecific emission model. This emission model was calibrated against the MOBILE model, a current industry standard for estimating vehicle emissions.

\section{B. Paper Organization}

The first part of the paper identifies and briefly describes several types of dispersion models. This description identifies the inputs required by the models and their functional characteristics. The microscopic, drive mode elemental vehicle emissions that is incorporated within the INTEGRATION traffic simulation model is then discussed.

Subsequently, an assessment methodology framework is described in which the INTEGRATION model is used to provide either point, line and area emission loads to environmental dispersion models. The capabilities of the INTEGRATION model in providing traffic measures of performance (MOP) and emissions estimates are then demonstrated through the application of the model to a network representation of Orlando, Florida. Finally, conclusions are made regarding the suitability of the proposed assessment methodology and the desired future model's functional capabilities. 


\section{Plume DisPERSION MODELING}

An increasing concern of traffic engineers and transportation engineers is the monitoring and control of air-borne traffic pollutants [1]. In the United States, this focus has been intensified through the passage of the Clean Air Act Amendments in 1990 and the Intermodal Transportation Efficiency Act of 1991. These Acts link federal funding for highway projects with the successful demonstration that the proposed projects will meet the legislated emission requirements. Therefore, independent of any standard assessments of the cost/benefit of a project, a procedure must be utilized which allows for the evaluation of the expected pollutant levels associated with the subsequent traffic operations.

The standard approach to solving this problem is to utilize Air Quality Standards (AQS) models, which calculate the resultant downwind concentrations for a set of the emission inputs over a given area of study. The dispersed concentration of each pollutant is computed by considering several factors, including meteorological transport and diffusion parameters, chemical transformations and removal processes. While much attention has been paid to this dispersion modelling, the significance of the emissions input cannot be underestimated, as it has proven in many cases to be the most critical element [2].

Most AQS models can be loosely divided into two categories based on the resolution of their modelling scale. Urban scale models examine the dispersion impacts for regions in the order of magnitude of 1 to $25 \mathrm{~km}$ in diameter, while local scale models examine pollutant dispersion in much smaller regions ranging from 10 to $1000 \mathrm{~m}$

\section{A. Urban Scale Dispersion Models}

In urban scale regions there may exist thousands, or possibly millions, of individual pollutant point sources. The modelling of individual sources independently has to date proven to be not only inefficient, but also impractical [3]. The common practice is therefore to combine the majority of the smaller pollutant sources, such as individual vehicles, with larger point sources such as factory smoke stacks, in order to develop larger area combined input sources. Specifically, multi-box, or Eulerain models have been developed to model the dispersion of these area sources [2].

In their simplest form, multi-box models overlay a series of square grids or boxes over the horizontal surface area to be considered. Depending on the particular model, the ceiling is then selected, often as a function of the mixing height. The model then calculates the change in concentration of the pollutant levels in the box during each time period $\Delta t$. This calculation is performed by considering both the net flow of pollutant through the sides, as defined by meteorological inputs, and by emissions entered directly into the box. This process can be further refined by allowing for variable horizontal resolution, so as to reflect the relative strengths of different emission inputs. Larger grids may be selected for low emission areas and smaller grids for larger emission areas, such as busy intersections and malls. This refinement can be expected to improve both the computational time and accuracy of traditional multi-box models with respect to traffic inputs [4].

\section{B. Local Scale Dispersion Models}

While the box model is effective at predicting the background levels of pollutants on a urban scale, special attention often must be paid to unique local effects. Studies [3,5] indicate that areas within 300 to $400 \mathrm{~m}$ of a roadway often experience pollutant levels which are significantly higher than those of the background levels predicted by urban scale models. Furthermore, these areas are often the most populated and contain the greatest number of pedestrians.

To address this special situation, micro-scale or local scale models are applied. These local scale models, in turn, are divided into two classes, freeway oriented models and urban canyon models.

Freeway Models- Freeway oriented models are used in areas where there are very few surrounding structures of significant height. They have traditionally been modelled using the Gaussian equation for an infinite line source.

This approach, however, has been criticised as being inaccurate [6], as it does not take into account the spatial distribution of emission levels along the roadway that result from the varying speed profiles. This variation is particularly pronounced at bottlenecks and busy interchanges which experience proportionally larger amounts of acceleration, deceleration and idling.

One proposed solution, to address this inaccuracy, has been to model discrete vehicles as point source emissions [6] in combination with the standard Gaussian equation for point sources. To this end, a number of micro-scale traffic models, such as NETSIM, have been utilized to generate the necessary discrete emissions inputs. While more accurate than the infinite line source approach, the point source method has to date been generally discounted as a result of the greater computational requirements [6].

Urban Canyon Models- The modelling of localized traffic effects is even more difficult in densely populated centres. In these areas, tall structures often exist to either side of the street creating urban canyon effects. In these situations, the dynamic effects of the nearby buildings on the pollutant dispersion must be examined. The most common approach, which was developed by Johnson [2], treats the urban canyon as a box model with varying concentrations on the windward and leeward sides of the traffic lanes [5]. 
The emissions inputs for this approach are generated by treating the roadway as an infinite line source, as with freeway models. However, concern has arisen regarding the use of line source emissions in this context as urban areas, in particular, are subject to significant temporal and spatial variations in emissions concentrations. Unfortunately, as a result of the complexities introduced by the building aerodynamics, no approach has yet been developed to treat the urban canyon model as a series of point sources [6].

\section{EMISSIONS SOURCE MODELING}

The accepted standard in North America, for assessing the emission impacts of transportation planning or traffic engineering proposals, is mobile source emission models. One such model is MOBILE [7], a program developed by the United States Environmental Protection Agency (EPA). Typically, emission factors are derived on the basis of average speed and are multiplied by an aggregate measure of network loading, namely vehicle miles traveled (VMT).

\section{A. Limitations of Source Emission Models}

Although these mobile source emission models are very detailed in terms of vehicle characteristics and operating conditions, they are much less able to respond to the detailed manner in which traffic management measures may influence the precise driving cycles that a particular vehicle experiences in completing its trip. The reason for this is that these models were primarily developed to assess vehicle fleet impacts rather than traffic management impacts. For example, MOBILE cannot consider a differential emissions impact when the same vehicle makes several short stops versus one long stop of equivalent duration. Similarly, MOBILE does not recognize that diversion to a longer, but faster, alternative route can be more environmentally efficient depending upon the distance and flow conditions experienced.

In order to better capture the environmental impacts of different potential traffic management measures, such as improved arterial signal coordination or reduced freeway congestion, an emission model which monitors individual vehicle movements must be utilized. Unfortunately, the availability of such microscopic emission models has to date been quite limited, as previous efforts to collect vehicle emissions in the field have proven to be extremely difficult and costly [8]. Furthermore, the success of incorporating any collected microscopic data into a form conducive to integration with transportation models has also been limited.

As a result, a mode elemental emissions model was developed and incorporated into the INTEGRATION traffic simulation model. A complete description of this model development may be found elsewhere in the literature $[9,10]$ and briefly summarized below.

\section{B. Description of Source Emission Models}

In order to better capture the relationship, between vehicle emissions and traffic flow/control characteristics, a set of emission models were developed on the basis of strategically selected outputs from the MOBILE model. These derived models estimate the emissions of individual vehicles as a function of the traffic flow characteristics and management strategies associated with the driven route.

Specifically, three driving modes are captured by a coupled elemental fuel consumption model and emission model, that considers idling, cruising at constant speed and acceleration/deceleration maneuvers. The fuel consumption model is linked to a series of equations which predict the quantity of hydrocarbon $(\mathrm{HC})$, carbon monoxide $(\mathrm{CO})$ and oxides of nitrogen $\left(\mathrm{NO}_{\mathrm{x}}\right)$ for a given volume of fuel consumed. The above emission and fuel consumption estimates are sensitive to the ambient air temperature and each individual vehicles speed profile, as shown for $\mathrm{CO}$ in Fig. 1. Furthermore, the estimates are also sensitive to the presence of engine cold starts, an effect which dissipates as the vehicle is driven. This factor dominates vehicle emissions at the start of a trip, as indicated for CO in Fig. 2.

The derived emission models were validated by comparing the results offered by the MOBILE standard and the proposed equations for comparable scenarios. This comparison yielded a correlation coefficient $\left(r^{2}\right)$ of approximately 90 percent. A complete description of the

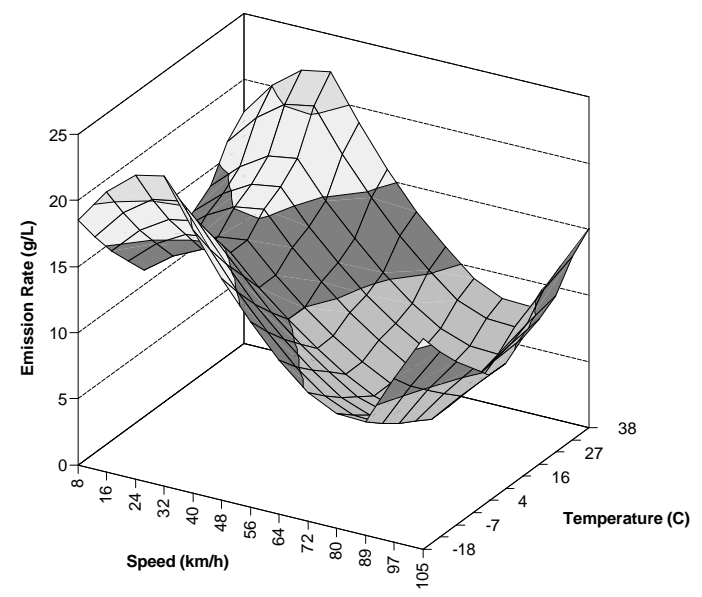

Fig. 1: Relationship between $\mathrm{CO}$ emissions per liter of fuel and speed/temperature. 


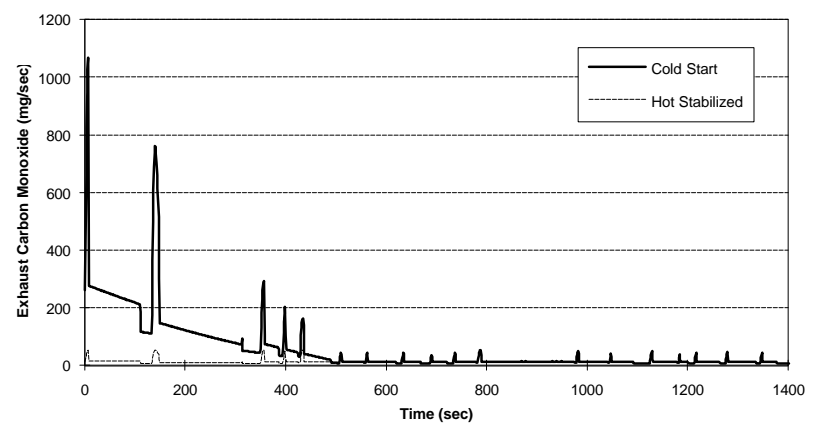

Fig. 2: Instantaneous $\mathrm{CO}$ emission profile of the EPA city driving cycle for a typical vehicle at $21^{\circ} \mathrm{C}$

calibration findings can be can be found in elsewhere in the literature [11], while the use of this emissions model in INTEGRATION is presented next.

\section{Description of INTEGRATION model}

Detailed descriptions of the INTEGRATION model, its input requirements, and other applications of the model are available in the literature $[12,13,14,15]$. The following section briefly describes those features relevant to the estimation of emissions and the evaluation of ATIS/ATMS.

The INTEGRATION model is a microscopic routingoriented simulation model of integrated freeway and surface street networks. The model is microscopic in the sense that individual vehicle movements are traced through the network as they stop at traffic signals or slow down within freeway queues, as a function of the prevailing ATMS strategy. In addition, each vehicle's identity is retained, as it travels through the network, in order to route each such vehicle based on its unique trip origin, destination, departure time and vehicle type. The tracking of each vehicle on a second-by-second basis permits the changes in $\mathrm{HC}, \mathrm{CO}$ and $\mathrm{NO}_{\mathrm{x}}$ to also be tracked each second as a function of the vehicless speed, acceleration/deceleration profile, and the extent to which the vehicles engine has warmed up. Such tracking not only permits accurate estimates of the magnitude of these quantities over time, but also permits the model to $\log$ the exact $\mathrm{X}$ and $\mathrm{Y}$ coordinate of where these emissions occur. These emissions can optionally be aggregated by vehicle, link, route, or horizontal grid cell within an urban area, as will be illustrated in Section V.

The model is also routing-based, in that only a vehicle's trip origin, destination and departure times are specified external to the model. This leaves the actual trip path and the arrival times at each link along the path to be derived within the simulation on the basis of the modeled interactions with any other vehicles. The actual path taken by a vehicle depends on the vehicles ATIS capability.

The ability to concurrently represent ATMS/ATIS impacts, and to provide second-by-second individual vehicle position by coordinate, yields a significant opportunity to perform environmental assessment of ATMS/ATIS strategies, as discussed next.

\section{ASSESSMENT METHODOLOGY}

The comprehensive evaluation of the impact of alternate ATMS/ATIS on traffic network performance and environment measures requires a flexible yet unified assessment framework. This framework should prescribe an assessment methodology that results in a level of accuracy in the estimation of traffic and environmental impacts that addresses the increasing legislative needs.

Typically, plume dispersion models have been utilized by environmental specialists to estimate the air quality impacts of various point, line, and area sources. Many of these models have evolved to a high level of complexity and have been shown to be useful in predicting air quality. Unfortunately, the level of accuracy of the inputs to these models has, in general, not kept pace. Thus, there exists a need for a method of accurately determining the levels of point, line and area emission sources for use as inputs to the plume dispersion models. Fig. 3 illustrates the proposed assessment framework, in which the INTEGRATION simulation model is used to provide estimates of the emission sources for input to the appropriate plume dispersion model.

\section{A. Traffic Simulation Component}

The traffic simulation component involves the modeling of the temporal and spatial dynamics of an entire peak period. Such simulation should first be performed for a base case in the absence of ATMS/ATIS, and then be repeated for alternative ATMS/ATIS deployments. While the simulation model can provide estimates of point source emissions for each vehicle in the network every second, such a data stream is likely too detailed for even the most advanced atmospheric dispersion models. Consequently, INTEGRATION permits a series of alternative data aggregations. One level of aggregation provides line source emissions for each network link per unit of time, say every 1 minute. An alternative level of aggregation yields minute-by-minute source emissions for a horizontal grid network, where each vehicles second-bysecond emissions are allocated to the cell grid corresponding to the current $\mathrm{X}$ and $\mathrm{Y}$ coordinate of the vehicle. A third level of aggregation permits a total source emission statistic to be generated for the entire urban area, say every 15 minutes. 


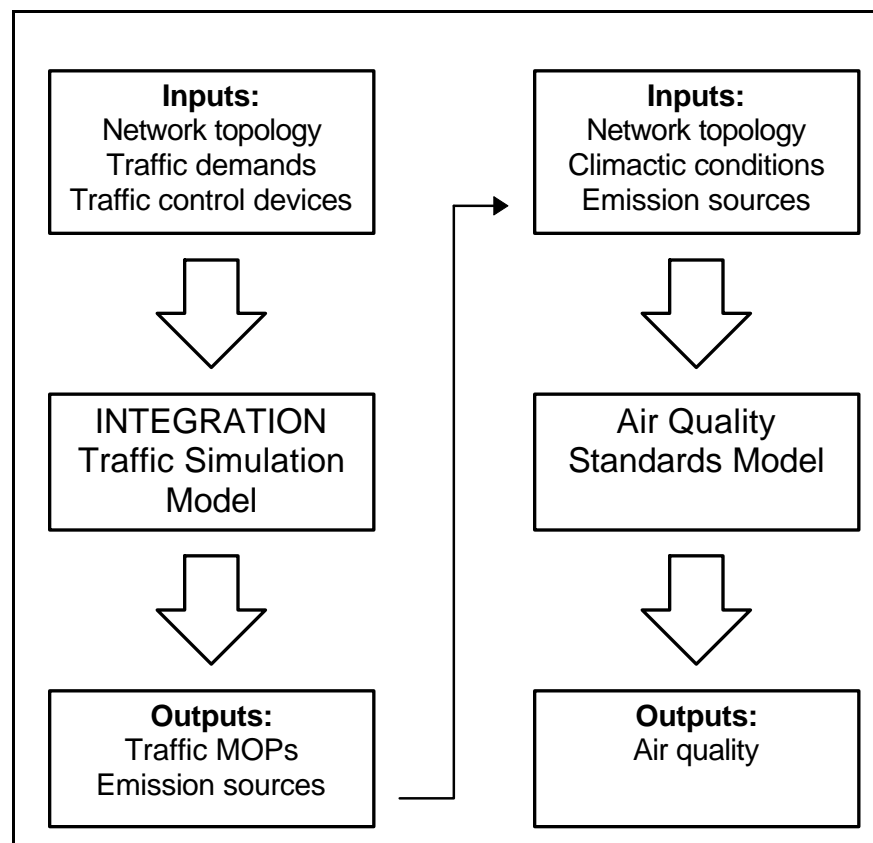

Fig. 3: Proposed air quality assessment framework

\section{B. Dispersion Model Component}

The above traffic simulation outputs permit either a very local canyon effect model to be executed based on the second-by-second vehicle point source generations, or based on the time series of link oriented line source generations. Alternatively, more large scale models can be driven by the grid based emission time series, where the dispersion from one grid cell to another can be modeled. Finally, the total emission output for the entire simulated area may be utilized as a single source emission input for a much larger area analysis considering many other sources of air pollution.

\section{Linking of Methodology Components}

The full impact of linking the above components is only realized when actual case studies are performed. For example, gating of traffic may result in the accumulation of queues in areas with more favourable atmospheric conditions. Alternatively, diversions may move source emissions to a cell where prevailing winds simply return the emissions to the location of the facility on which the original congestion was present. Finally, the detailed spatial and temporal interactions that can now be modelled permit both emissions equity and peaking to be considered, as well as the impact of exposure severity and duration.

\section{SAMPle Methodology APPliCATION}

It is anticipated that the proposed linked methodology will create the possibility to perform a range of assessments well beyond those originally envisioned by the methodology developers. Consequently, the following sample application simply illustrates but a small sample of the types of analyses that now may be possible.

\section{A. Network and Fleet Description}

To demonstrate the suitability of applying the INTEGRATION model to realistic networks, the model was applied to Orlando, Florida. The network, illustrated in Fig. 4, covers approximately $120 \mathrm{~km}^{2}$ in Orlando, Florida. The traffic network consists of 2,669 links, 71 origin/destination zones, and 1,307 intermediate nodes. The network includes Interstate 4 (thick line running north-south in Fig. 4) and most major arterials. Local streets are not included.

Two hours of the p.m. peak period (from 4-6 p.m.) were modelled. The QueensOD synthetic origin-destination traffic demand estimation model $[16,17]$ was used to generate time varying demands from observed loop detector link flows [18,19]. As a result, a total of 62,899 vehicles were loaded onto the network during the two hours. The simulation model was run for an additional hour to permit all vehicles to clear the network. The ambient air temperature was set to be $32^{\circ} \mathrm{C}$, with the result that cold start impacts on emissions were negligible. The vehicle population was assumed to be comprised of mid-sized vehicles have standard EPA fuel ratings of $11.8 \mathrm{l} / 100 \mathrm{~km}$ for city driving and $8.9 \mathrm{l} / 100 \mathrm{~km}$ for highway driving.

\section{B. Sample Model Results for a Network}

Sample traffic and environmental MOPs were produced at 15 minute intervals. A three-dimensional matrix of area source emissions was produced for each emission $\mathrm{HC}, \mathrm{CO}$ and $\mathrm{NO}_{\mathrm{x}}$. Each matrix consisted of 10 columns and 48 rows, where each row and column represented $1 / 2 \mathrm{~km}$ distance increments along the $\mathrm{X}$ and $\mathrm{Y}$ directions in the network, respectively. The third dimension of each matrix consisted of 12 layers, one layer for each 15 minutes of simulation. These matrices provide a dynamic representation of the emission sources throughout the entire spatially distributed network.

Fig. 5 depicts the spatially distributed $\mathrm{CO}$ emissions accumulated between 4:45 and 5:00 p.m. The contours clearly indicate that the I-4 dominates the network as a source of $\mathrm{CO}$ emissions. It is further evident that the southern portion $(<12 \mathrm{~km})$ of the I-4 is a greater contributor than the northern portion $(>12 \mathrm{~km})$. Above average emission levels at certain critical congested locations can also be noted. Similar contours were generated for $\mathrm{HC}$ and $\mathrm{NO}_{\mathrm{x}}$ emissions, but are not presented here.

It is anticipated that these three-dimensional matrices may be directly utilized as input to area or box AQS models. In the event that more detailed emission source data were desired for a particular portion of the entire network, line source emission 
profiles should be generated for a specific route, as illustrated next.

\section{Sample Model Results for a Complete Facility}

Consider Fig. 6, which depicts the temporal variation in $\mathrm{CO}$ emissions along a portion of the eastbound direction of the I-4. Station numbers are located at approximately $1 / 2 \mathrm{~km}$ intervals. These data may be used to analyze the air quality within a specific area, with a greater level of detail than might be achieved using an area-wide base model. These data may also be compared to traffic MOPs such as speed (Fig. 7), in order to gain an understanding of the impacts that ATMS, such as ramp metering or incident management may have on both traffic versus air quality MOPs.

Traffic and air quality MOPs may also be obtained using the INTEGRATION model at a resolution of an individual link. For

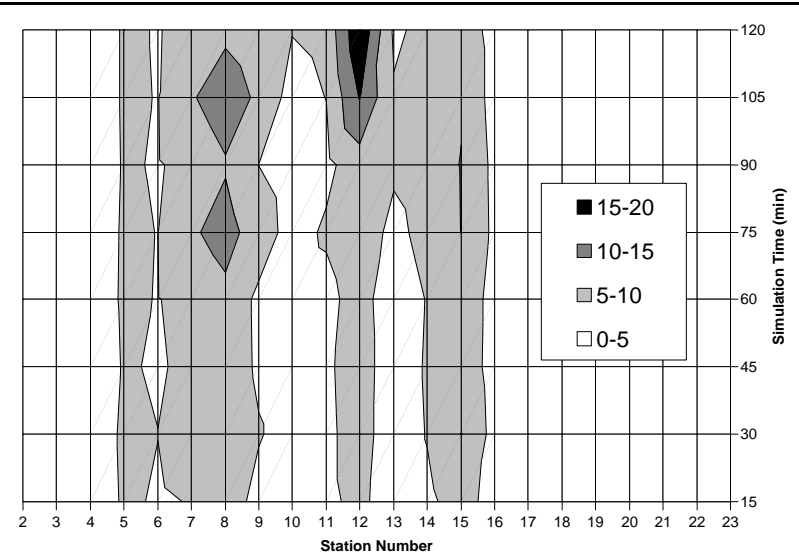

Fig. 6: Temporally distributed CO emissions (kg) for the I-4 eastbound in Orlando, Florida

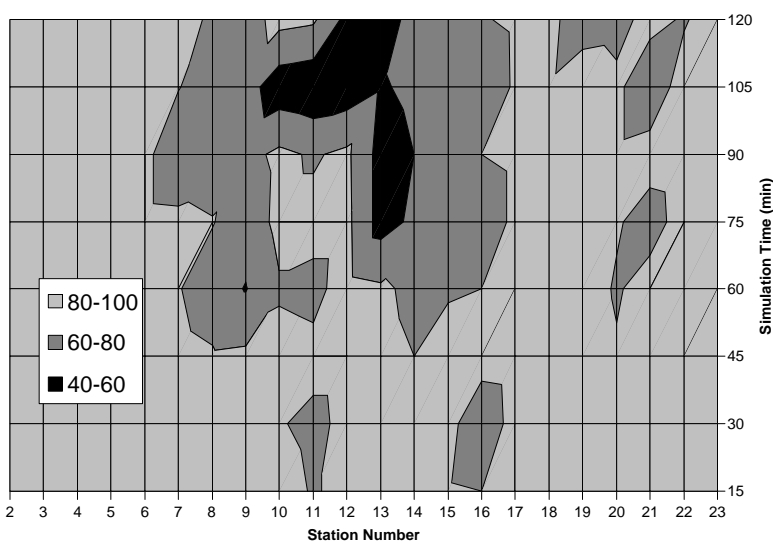

Fig. 7: Temporally distributed speeds $(\mathrm{km} / \mathrm{h})$ for the I-4 eastbound in Orlando, Florida

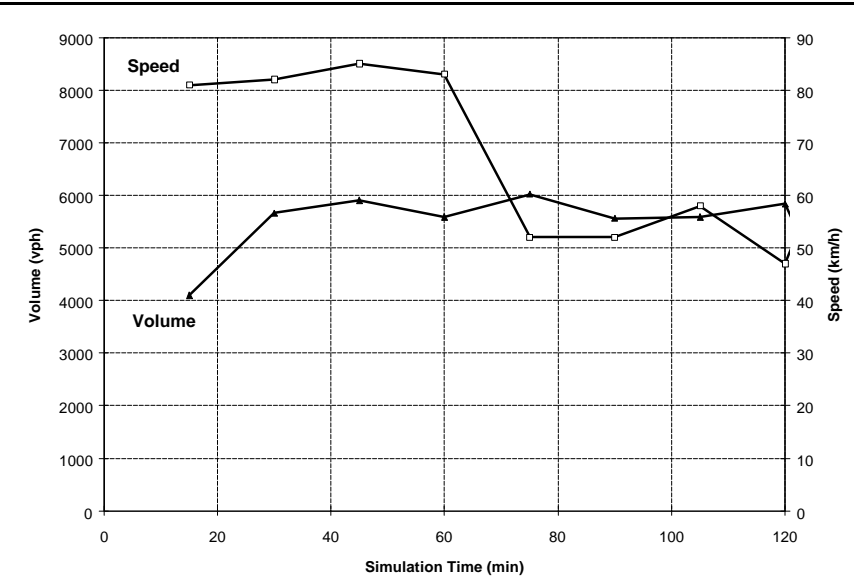

Fig. 8: Time series of volume (vph) and speed $(\mathrm{km} / \mathrm{h})$ estimates for a single I-4 link

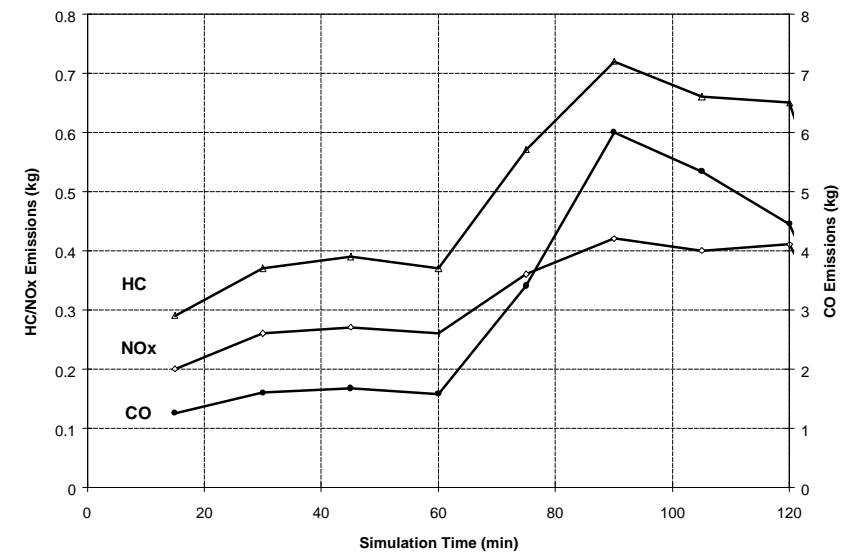

Fig. 9: Time series of $\mathrm{HC}, \mathrm{CO}$, and $\mathrm{NO}_{\mathrm{x}}$ emission estimates $(\mathrm{kg})$ for a single I-4 link

example, Fig. 8 and Fig. 9 depict temporal traffic and air quality MOPs, respectively for a single link on the I-4. These data may be used as inputs to AQS models when a high resolution of local emission sources is required.

It may be noted that $\mathrm{NO}_{\mathrm{x}}$ emissions, which should be lower in Fig. 9 due to the speed reduction indicated in Fig. 8, actually increased due to the increase in traffic density, which was not noted explicitly in Fig. 8.

\section{CONCLUSIONS}

Current legislation makes federal funding for certain highway projects contingent on the successful demonstration that specified AQS be met. Current legislation also defines a schedule in which increasingly restrictive AQS will be enforced over the next decade. Achieving these AQS will require the innovative and effective use of ATIS/ATMS strategies. The 
[8] S. Cadle, R. Gorse, and D. Lawson, "Real-world vehicle emissions: a summary of the third annual CRC-APRAC on-road vehicle emissions workshop," in Air and Waste, vol. 43, pp. 1084-1090, August 1993.

[9] M. Baker, "Fuel consumption and emission models for evaluating traffic control and route guidance strategies," Masters Thesis, Department of Civil Engineering, Queen's University, Kingston, Canada, 1994.

[10] M. Van Aerde, and M. Baker, "Modeling fuel consumption and vehicle emissions for the TravTek system," VNIS Annual Conference, Ottawa, Canada, 1993.

[11] M. Baker, and M. Van Aerde, "Microscopic simulation of EPA fuel and emission rates for conducting IVHS assessments," IVHS America Annual Conference Proceedings, Washington, DC, 1995.

[12] M. Van Aerde, "INTEGRATION: a model for simulating integrated traffic networks. User's guide for model version $1.5 \mathrm{~g}$ and Associates, Ltd. and Transportation Systems Research Group, Queen's University, Kingston, Canada, 1994.

[13] H. Rakha, M. Van Aerde, E.R. Case, and A. Ugge, "Evaluating the benefits and interactions of route guidance and traffic control strategies using simulation," VNIS Annual Conference, Toronto, Canada, 1989.

[14] K. Wunderlich, M. Van Aerde, B. Hellinga, and J. Harding, "Macro-level traffic simulation and case study development for IVHS system architecture evaluation," IVHS America Annual Conference, Washington, DC, 1995.

[15] B. Hellinga, and M. Van Aerde, "An overview of a simulation study of the Highway 401 freeway traffic management system," Canadian Journal of Civil Engineering, vol. 21, pp.439-454, 1994.

[16] B. Hellinga, "QueensOD: a model for estimating static and dynamic origin-destination demands from observed link flows. User's guide for model version 1.3a., M. Van Aerde and Associates, Ltd. and Transportation Systems Research Group, Queen's University, Kingston, Canada, 1994

[17] B. Hellinga, "Estimating dynamic origin-destination demands from link and probe counts," Ph.D. Thesis, Department of Civil Engineering, Queen's University, Kingston, Canada, 1994.

[18] M. Van Aerde M., H. Rakha, M. Baker, L. Rilett and V. Inman, Simulation Based Estimates of Benefits to TravTek Drivers and Other Network Users, Forthcoming IVHS Journal Special Issue on TravTek, 1995.

[19] M. Van Aerde, and H. Rakha, TravTek Evaluation Task E $E_{I}$ - Modeling Study, Technical Report FHWA-RD-95-090, 1995. 


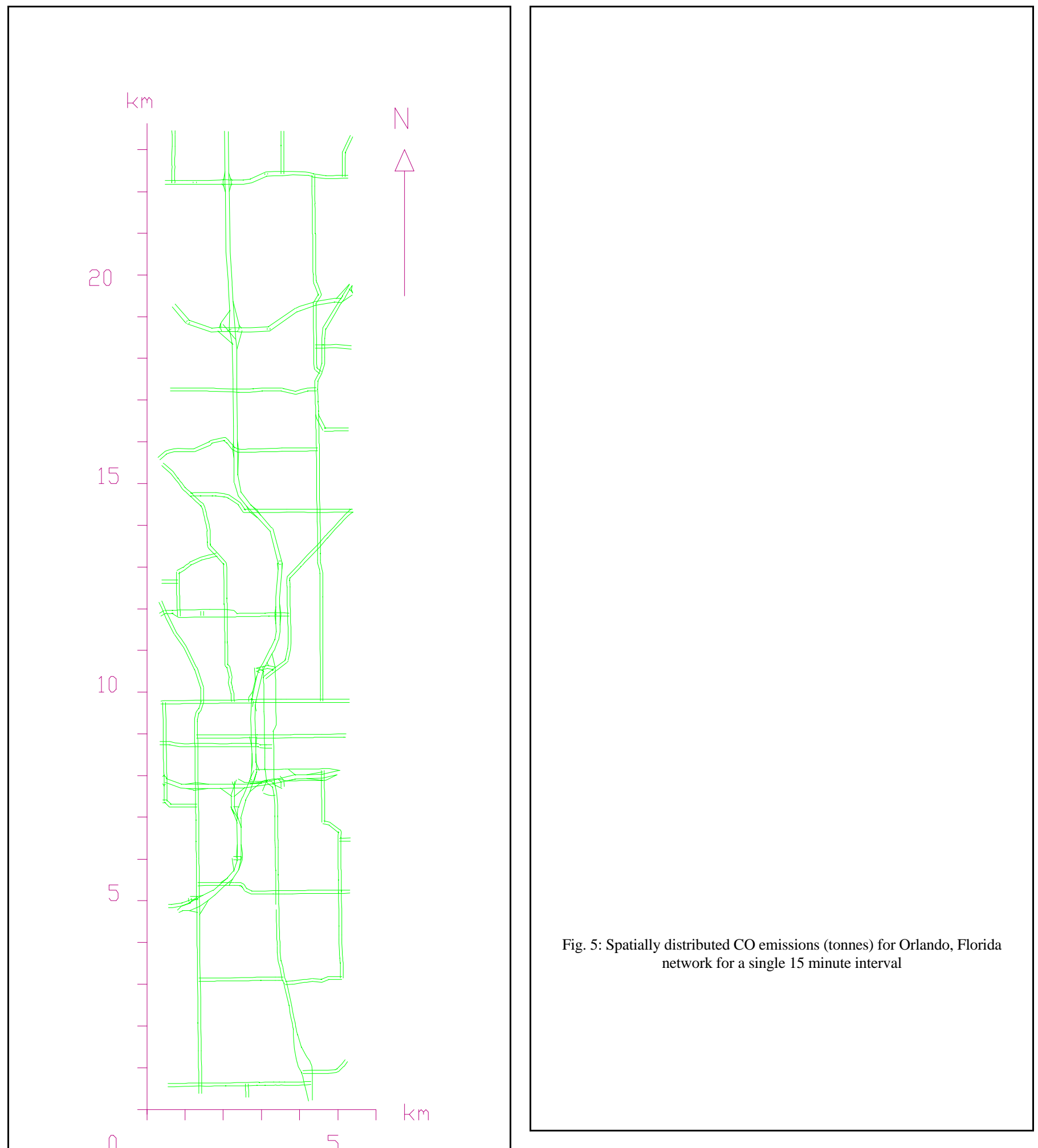

Fig. 4: Orlando, Florida traffic network 